\title{
Gegen die Sinuskurve - Zur Theorie des Konjunkturzyklus und seiner Darstellung in österreichischen Schulbüchern
}

stefan_hinsch@yahoo.de, Abendgymnasium Wien

eingereicht am: 10.02.2016, akzeptiert am: 23.04.2016

\begin{abstract}
Die in österreichischen Schulbüchern übliche Darstellung des Konjunkturverlaufs als Zyklus (mit der Graphik einer Sinuskurve) suggeriert eine regelmäßig um einen Gleichgewichtszustand (oder einen stabilen Wachstumspfad) schwankende Wirtschaftsentwicklung. Eine solche Herangehensweise kann aber der Weltwirtschaft nach dem Kriseneinbruch von 2007/08, die das Wachstumsparadigma der Nachkriegszeit in Frage stellt, nicht gerecht werden. Der Artikel problematisiert Zyklustheorien der Konjunktur, analysiert die Darstellung in österreichischen Schulbüchern und schließt mit Vorschlägen für Alternativen in der Unterrichtsgestaltung.
\end{abstract}

Keywords: Konjunkturtheorie, Krise, Wirtschaftsdidaktik, Schulbücher

\section{Against the sine curve. On the theory of the economic cycle and its representation in Austrian text- books}

The discussion of business activity as a regular cycle (represented by a sine curve) in Austrian textbooks suggests an economy oscillating periodically around an equilibrium (or a stable growth path). This approach is unable to grasp the world economy after the crisis of 2007/08, which compromises the growth paradigm of post-war economic development. This article deals with the theory of the business cycle, analyses its representation in Austrian textbooks and offers suggestions for an altered approach on the didactics of the business cycle.

Keywords: business cycle, crisis, economic didactics, text books

Eröffnet sei mit einer Anekdote, die der Auslöser für diesen Artikel war. Bei einem Matura-Prüfungsgespräch im Jahr 2014 wurde die Kandidatin durch den prüfenden Lehrer gefragt, was denn nach einer Rezession komme. Diese Frage wurde mit „die Depression“ zur Zufriedenheit des Prüfers beantwortet, der aber weiterfragte: „Und dann?“ Die scheinbar wieder richtige Antwort lautete „der Aufschwung“. Die Prüfung wurde positiv bewertet, der Inhalt bleibt problematisch.

Denn glücklicherweise folgt nicht auf jede Rezession eine Depression. Und auf eine Depression folgt nicht einfach ein neuer Aufschwung. Es sei hier keinem ökonomischen Determinismus das Wort geredet, aber auf die letzte große Depression folgten Diktatur und Vernichtung, ein entsetzlicher Krieg, schließlich ein umfassender Paradigmenwechsel in der Wirtschaftstheorie und der Wirtschaftspolitik - und dann gab es einen neuen Aufschwung. Als Ergebnis der aktuellen griechischen Depression scheint eine weitgehende Peripherisierung durchaus denkbar. Und die Depression in Südeuropa stellt die europäische Integration in Frage. Ein neuer Aufschwung durch die Kraft des Zyklus ist nicht absehbar (vgl. Hinsch \& Langthaler 2016).

Nur wie kommen solche problematischen Inhalte in eine Maturaprüfung? Denn seit dem Kriseneinbruch der Jahre 2007 / 2008 kommt der Beschäftigung mit Konjunktur und Depression im GW-Unterricht schließlich erhöhte Bedeutung zu. Der Schluss liegt nahe, dass Schulbuchdarstellungen von Konjunktur wenigstens eine gewisse Verantwortung tragen. Für diesen Beitrag werden daher österreichische GW-Büchern für die Sekundarstufe II der AHS untersucht.

Spezifischer beschäftigt sich dieser Artikel aber mit den Theorien des Konjunkturzyklus und deren Verarbeitung in Schulbüchern. Denn Konjunktur (die allgemein nur die momentane wirtschaftliche Lage 
bezeichnet) wird in den untersuchten Schulbüchern als Konjunkturzyklus gefasst. Dafür gibt es keine zwingende Notwendigkeit: Die Tatsache, dass die aktuelle Lage einer Gesamtwirtschaft Schwankungen unterworfen ist, impliziert nicht automatisch einen Zyklus. Bewusst oder unbewusst wird damit auf $\mathrm{Zy}$ klustheorien rekurriert, die seit den 1960er Jahren an Bedeutung verloren haben und deren letzte wichtigere Formulierung Anfang der 1980er Jahre stattfand. Im Zentrum dieser Theorien steht eine Abfolge wirtschaftlicher Phasen, häufig dargestellt als Sinuskurve, und eine regelmäßig um einen Gleichgewichtszustand (oder einen stabilen Wachstumspfad) schwankende Wirtschaftsentwicklung. Den Zyklustheorien geht es nicht in erster Linie um Wirtschaftskrise, sondern um allgemeine Gesetze kapitalistischer Entwicklung - die zusätzlich in eine quasi-naturwissenschaftlichen Form gefasst werden und sich dem Einfluss handelnder Menschen weitgehend entziehen. Der Nachweis für diese Behauptung wird in diesem Artikel versucht.

Als besondere didaktische und inhaltliche Probleme ergeben sich: Aus der Perspektive einer Wissenschaftsorientierung erfolgt ein Rückgriff auf Theorien, die heute wenig Bedeutung haben. Aus der Perspektive einer Orientierung an den Wirtschaftsbürgerinnen und Wirtschaftsbürgern ermöglichen die Schulbuchdarstellungen zu wenig Auseinandersetzung mit der nach 2007/08 eingeleiteten Krisenpolitik - zumindest wird der Rahmen für diese Auseinandersetzung in problematischer Weise abgesteckt. Und aus der Perspektive einer Orientierung an der Lebenswelt kann berechtigten Ängsten und Sorgen um die Entwicklung von Arbeitsverhältnissen und Arbeitslosigkeit nur unvollständig Raum gegeben werden.

Dieser Artikel versucht zu zeigen, dass die Theorien des Konjunkturzyklus mehr bedeuten als nur die Beschäftigung mit der Wirtschaftslage. In der Folge wird die Darstellung von Konjunktur in österreichischen Schulbüchern für die Sekundarstufe II analysiert und untersucht, welche Elemente der Zyklustheorien hier zu finden sind, um danach die Unzulänglichkeit dieser Herangehensweise vor dem Hintergrund der Entwicklung der Weltwirtschaft und der aktuellen volkswirtschaftlichen Debatte nachzuzeichnen. Schließlich sollen vorsichtig Alternativen für die Unterrichtspraxis aufgezeigt werden.

Nicht behandelt werden Theorien zur Erklärung der aktuellen Weltwirtschaftskrise, und es wird auch kein Versuch unternommen diese zu didaktisieren. Stattdessen geht es um die Vorbedingung einer solchen Fragestellung: Denn um das Augenmerk auf krisenhafte Entwicklungen werfen zu können, bedarf es eines Bruchs mit der didaktischen Darstellung von Konjunktur als regelmäßigem Zyklus.

\section{Die Theorie des Konjunkturzyklus}

Eine Zusammenschau von 150-200 Jahren Konjunkturtheorie ist nicht möglich, aber es sollen die wichtigsten Ansätze herausgegriffen werden, um die Diskussionslinien zu erkennen.

Wenn man zur Definition ein etwas neueres deutsches Standardwerk bemüht, dann wird der Konjunkturzyklus von dem neoklassischen Zyklustheoretiker Alfred Maußner als „wiederkehrende wirtschaftliche Wechsellagen industrialisierter Marktwirtschaften " gefasst. Danach schwankt die „wirtschaftliche Aktivität wellenförmig um ihren Normalzustand“ (Maußner 1994, 2 f.). Dabei werden vier Phasen mit Abschwung (recession), Konjunkturtal (depression), Aufschwung und Hochkonjunktur abgegrenzt (ebd., 3). Bereits in der einflussreichen Arbeit von Joseph Schumpeter (1883-1950) über den Konjunkturzyklus finden sich „Prosperity, Recession, Depression” und „Revival' (Schumpeter 1939, 155), allerdings mit der Einschränkung "While recession and-if depression occurs-revival are necessary parts of the cyclical process of economic evolution, depression itself is not" (ebd., 157). Schon diese Definitionen zeigen: Die Theorien des Konjunkturzyklus benennen nicht einfach Konjunkturzustände (Rezession, Depression oder Hochkonjunktur), sie postulieren Regelmäßigkeit über wellenförmige Schwankungen, wiederkehrende Zustände und notwendige Phasen ökonomischer Entwicklung.

Als erster Theoretiker wirtschaftlicher Krisen aber auch des Konjunkturzyklus wird in der Regel Sismonde de Sismondi (1773-1842) genannt. Seine Theorien von Ungleichverteilung und Unterkonsum weisen auf eine Krisenhaftigkeit kapitalistischer Entwicklung hin, jedoch postuliert er kein zyklisches Schwanken um einen Normalzustand oder makroökonomisches Gleichgewicht, sondern steht der Idee des Gleichgewichts insgesamt skeptisch gegenüber (vgl. Sowell 2006, 104 ff.).

Eine Formulierung eines ökonomischen Zyklus findet sich hingegen bei Clément Juglar (1819-1905). Sein Konjunkturzyklus geht als ,fixed-investment cycle“ in die Literatur ein, in dem Phasen schneller Investitionen zu Überkapazitäten führen; begründet wird das Ganze aber eher im Rahmen einer psychologischen Konjunkturtheorie, in der die menschliche Natur zu einem Exzess an Risikobereitschaft und Spekulation führt. Für Juglar steht aber nicht die Begründung von Wirtschaftskrise im Vordergrund, sondern der Nachweis ihrer Regelmäßigkeit - eben der Zyklus (vgl. Besomi 2009).

Auf Juglar folgen weitere Theorien des Zyklus, bekannt werden Nikolai Kontradieff (1892-1938) und Joseph Kitchin (1861-1932). Kontradieff, Juglar und Kitchin verdanken einen Teil ihres Ruhmes 
Joseph Schumpeter, der ihre Zyklen als sich überlagernde Wellen darstellt (Schumpeter 1939, 209 ff.). Graphisch taucht hier auch das Motiv der Sinuskurve auf (ebd., 211). Schumpeter liefert dabei eine eigenständige Begründung des Konjunkturzyklus, er sieht ihn als Reaktion und Anpassung auf die Erschütterung eines wirtschaftlichen Gleichgewichts durch Innovationen und unternehmerische Initiative. Wobei der Zyklus keine einfache Ableitung aus Innovation und Entrepreneur darstellt, sondern eine komplizierte Reaktion auf diese Kräfte, in der etwa auch monetäre Zusammenhänge und Psychologie eine Rolle spielen (ebd., 132 ff.).

Wieder gilt: Für Schumpeter ist der Konjunkturzyklus nicht in erster Linie eine Beschäftigung mit den Gründen von krisenhaften Entwicklungen, sondern - eigentlich eher im Gegenteil - eine Beschäftigung mit kapitalistischer Entwicklung im Allgemeinen. So sagt er über Juglar: „His great merit is that he pushed the crisis into the background and that he discovered below it another, much more fundamental, phenomenon, the mechanism of alternating prosperities and liquidations" (ebd., 170). Und: Im Schumpeterschen Zyklus werden Prosperität und Depression durch einen einheitlichen Prozess erklärt, „set into motion by a definite force or cause" (ebd. 148). Der "definite mechanism" (ebd., 148) des Konjunkturzyklus scheint auch für die gesamte kapitalistische Entwicklung gültig - in jedem Fall werden keine Einschränkungen bezüglich der historischen Periode einer Gültigkeit getroffen.

Auch in der Tradition des Keynesianismus spielt der Konjunkturzyklus in der Folge eine wichtige Rolle. Bei Keynes selbst finden sich Elemente der Theorie eines Zyklus, diese sind aber rudimentär und widersprüchlich. Für Keynes bedeutet eine zyklische Bewegung nicht nur "that upward and downward tendencies, once started, do not persist forever", sondern auch "that there is some recognisable degree of regularity in the time sequence and duration of the upward and downward movements." (Keynes 1936, 314) Diese Bewegung begründet er dann durch eine Schwankung des Grenzertrags des Kapitals (marginal efficiency of capital). In einer Boomphase nimmt diese ab, besonders in jenen Sektoren, die die meisten Investitionen angezogen haben. In der Krise führt dann ein Fallen des Kapitalstocks "through use, decay and obsolescence" (ebd., 318) zu einer zunehmenden Knappheit an Kapital und einem wieder steigenden Grenzertrag (ebd., 315 ff.).

Im Anschluss jedoch weist er die Idee einer allgemeinen Überinvestition für die allermeisten Fälle zurück, bezieht den sinkenden Grenzertrag nicht auf Kapitalstock und Investitionen in ihrer Gesamtheit, sondern auf überzogene Ertragserwartungen in einzelnen Sektoren - das Problem wird daher nicht als Überinvestition, sondern als Fehlinvestition gefasst.
Eine Fehlinvestition, „which sometimes happens, we may add, even if there is no boom " (ebd., 321). Der Krise wird dabei keine notwendige oder nützliche Funktion zugeschrieben, sie möge durch staatliche Maßnahmen bekämpft werden, um die Wirtschaft permanent in der Nähe eines Booms zu halten (ebd., 320 ff.).

Während aus der ersten Formulierung Keynes' ein regelmäßiger Zyklus abgeleitet werden kann, gilt das für die zweite Überlegung nicht mehr. Konjunkturzyklen in keynesianistischer Tradition lassen die Fehlinvestition daher unter den Tisch fallen. So mathematisiert John Hicks (1904-1989) 1950 einen Konjunkturzyklus, in dem Schwankungen der Investitionen die Gesamtnachfrage zwischen Vollbeschäftigung und Krise oszillieren lassen. Aber nicht durch Fehlinvestitionen, sondern weil die Nachfrage nach Investitionsgütern bei steigender Gesamtnachfrage und fehlenden Kapazitäten stark steigt, und bei fallender Gesamtnachfrage und Überkapazitäten zusammenbricht (Hicks 1950). Solche Überlegungen finden sich bei Überinvestitionstheorien schon vor Keynes, etwa bei Arthur Spiethoff (vgl. Kulla 1996, 112 f.).

1967 entwickelte Richard Goodwin (1913-1996) das Modell eines Konjunkturzyklus, das die Lohnquote im Zentrum hat: Geringe Lohnquoten erhöhen Unternehmensgewinne, diese lösen zusätzliche Investitionen aus, was die Gesamtnachfrage erhöht. Schließlich nähert sich die Wirtschaft der Vollbeschäftigung, dadurch steigt die Lohnquote, die Investitionen nehmen $a b$ und eine Krise wird eingeleitet - solange bis die Arbeitslosigkeit die Löhne wieder nach unten zwingt. Wegen der Bedeutung der Gesamtnachfrage und der klebrigen Preise und Löhne ist das Modell keynesianistisch beeinflusst - allerdings ein Keynesianismus des neoklassisch geprägten Nachkriegs-Mainstreams, in dem die Beschäftigung mit der Lohnhöhe fällt und höhere Löhne keinen positiven Effekt für die Gesamtnachfrage haben (vgl. Minsky 1986, 149 ff.). Gleichzeitig wird Goodwins Theorie durch Beschreibungen von Räuber-Beute-Beziehungen in der Biologie inspiriert, und auch die mathematischen Grundlagen werden der Biologie entlehnt. Goodwins Konjunkturzyklus weist daher Gemeinsamkeiten mit der Zyklizität der Entwicklung von Räuber- und Beutepopulationen im Tierreich auf. Es vermehren sich zuerst die Beutetiere, dann die Räuber, diese fressen so viele Beutetiere, dass erst die Population der Beute zurückgeht und zeitversetzt auch jene der Räuber - dadurch können sich die Beutetiere wieder stärker vermehren. Statt Räuber und Beute setzt Goodwin Bezieher von Kapitaleinkommen und Lohnbezieher, deren Konkurrenzkampf oszillierende Konjunkturschwingungen hervorbringt (vgl. Maußner 1994, 161 ff.). 
Es sei festgestellt: Während Schumpeters Konjunkturzyklus eine Art ewiger historischer Prozess wird, von den Menschen kaum mehr zu beeinflussen, wird dieser Prozess bei Goodwin zusätzlich naturalisiert.

Die neoklassische Tradition bringt mehrere Modelle, die zyklische Schwankungen der Wirtschaft erklären, der letzte und bekannteste Versuch erfolgt in den 1980er Jahren und ist dabei auch die letzte einflussreiche Formulierung einer Zyklustheorie: Plossners Real Business Cycles. In diesem neoklassischen Ansatz (es gelten rationale Erwartungen, perfekte Informationen und perfekte Märkte) führt der externe Schock nicht erwarteter Produktivitätssteigerungen zu einer Substitution von Arbeit durch Freizeit (Plossner 1989). Damit wird dem Konjunkturzyklus übrigens jedes Element einer echten Krisenanalyse genommen - eine Theorie, in der jede Arbeitslosigkeit freiwillig erfolgt (weil Freizeit vorgezogen wird) scheint das Elend einer Massenarbeitslosigkeit unzureichend abzubilden.

Ab den 1960er Jahren, mit dem Erstarken der Neoklassik in der neoklassisch-keynesianischen Synthese und dem etwas später folgenden Monetarismus ging das Interesse am Konjunkturzyklus zurück. Die Neoklassik ist eine Theorie des Gleichgewichts (die Real Business Cycles nehmen eine Sonderstellung ein und stellen die Theorien des Gleichgewichts auch nicht wirklich in Frage). Ausgehend von den Ideen von Léon Walras liefert die Neoklassik nach dem Zweiten Weltkrieg die mathematisierte Allgemeine Gleichgewichtstheorie, mit der demonstriert wird, dass eine dezentralisierte Marktwirtschaft ein kohärentes Resultat bringt (allerdings unter heroischen Annahmen) (vgl. Debreu 1959). Wirtschaftliche Verwerfungen sind dem Modell extern. So erklären Milton Friedman und Anna Schwartz die Große Depression der 1930er Jahre mit einem externen Schock der Geldmengenverknappung (Friedman 1963). Bis zum Ausbruch der Finanzkrise 2007/08 dominierten Konzepte, die die Stabilität einer möglichst deregulierten Marktwirtschaft betonten. Zentral sind dabei Überlegungen, die die Effizienz freier Finanzmärkte postulieren, oder Thesen, die niedrige Inflation als entscheidende Voraussetzung für Finanzmarktstabilität beschreiben (vgl. Bordo 1998; für eine kritische Betrachtung vgl. etwa Frydman 2011, 81 ff.). Langanhaltende erhöhte Arbeitslosigkeit wird dabei Marktrigiditäten zugeschrieben (vgl. Eichengreen 2007, 252 ff.), Rezessionen und Phasen der Überhitzung seit den 1960er Jahren werden in erster Linie mit einem Zuviel (Überhitzung), oder einem Zuwenig (Rezessionen) an Stimulus durch Geld- oder Fiskalpolitik erklärt (vgl. Plossner 1989, 51). Dieser Ansatz zum Verständnis wirtschaftlicher Einbrüche scheint für viele Situationen auch durchaus schlüssig, etwa für die europäische Rezession Anfang der 1990er Jahre (vgl. Eichengreen 2007, 358). Aber ein Zuviel oder
Zuwenig an Stimulus ist natürlich in einer nächsten Phase relativ leicht zu korrigieren. Das bedeutet: Über die Dosierung von Fiskal- und Geldpolitik in der Konjunkturpolitik wurde gestritten, aber in einer Synthese aus Milton Friedman und John Maynard Keynes hielt der volkswirtschaftliche Mainstream eine Katastrophe vergleichbar mit der Depression der 1930er Jahre für ausgeschlossen (Krugman 2009, 4). Damit können die Verwerfungen der Krise seit 2008 in der Folge nur schlecht abgebildet werden, in der ein gewaltiges Aus$\mathrm{maß}$ an geldpolitischem Stimulus (bei gleichzeitiger weitgehender fiskalpolitischer Enthaltsamkeit) bisher keine Normalisierung erreichen konnte (Für einen Überblick über die Notenbankpolitik siehe Kader \& Schwarzer 2015, 277 ff.).

\section{Konjunkturzyklus in österreichischen Schulbüchern für die Sekundarstufe II}

In der Folge werden fünf österreichische Schulbücher verglichen, untersucht wurde dabei jeweils der Abschnitt über Wirtschaftspolitik in der 11. Schulstufe in dessen Rahmen überall eine Auseinandersetzung mit Konjunktur stattfindet. Analysiert wurden: Durchblick 7 (Hofmann-Schneller et al. 2015), Geospots 7 bis 8 (Germ et al. 2013), Meridiane 7 (Hitz et al. 2013), klar_Geographie und Wirtschaftskunde 7 (Kaps 2011) sowie Raum Gesellschaft Wirtschaft 7 (Malcik \& Sitte 2013). Kriterium für die Auswahl war eine Neuauflage ab 2010, um einen zeitlichen Abstand zum Krisenausbruch 2007/2008 zu gewährleisten.

Alle untersuchten Schulbücher verschreiben sich dabei keiner bestimmten zyklischen Konjunkturtheorie. Wo Gründe für Konjunkturschwankungen behandelt werden, werden sie gegenübergestellt und überblicksmäßig beschrieben. In Durchblick 7 finden sich etwa Überinvestitionstheorie, Unterkonsumptionstheorie und Psychologische Konjunkturtheorie (wenn auch sehr klein am Rand) (Hofmann-Schneller et al. 2015, 54), in Meridiane 7 werden äußere Ursachen, fehlerhafte Erwartungen, Wirkungsverzögerung wirtschaftspolitischer Maßnahmen (in der Erklärung kommen dann allerdings Goodwins zu hohe Lohnabschlüsse, keine Wirtschaftspolitik) und Schwankungen der Kreditvergabe genannt (Hitz et al. 2013, 98). Teilweise wird darauf ganz verzichtet oder einleitend einige exogene Faktoren zur Erklärung von Konjunkturschwankungen verwendet, in RGW 7 etwa strenge Winter oder schwankende Ölpreise (Malcik \& Sitte 2015, 47). Ein typisches Kennzeichen zyklischer Konjunkturtheorien, Schwankungen der Wirtschaftsentwicklung letztlich einem einzelnen Faktor zuzuordnen, entfällt in allen analysierten Büchern. Dennoch findet sich die Rückbindung an Schumpeter und 
seinen ewigen Zyklus kapitalistischer Entwicklung: Durch die Darstellung der Konjunktur als Sinuskurve, sowie den regelhaften Ablauf verschiedener Phasen. Nicht in jedem Buch ist das gleich deutlich, aber es ist überall zu finden.

Die Darstellung des Konjunkturverlaufes als Sinuskurve findet sich in vier von fünf Büchern, lediglich Geospots 7 bis 8 (Germ et al. 2013) verzichtet darauf. In Meridiane 7 wird das Bild der Sinuskurve allerdings durch eine unmittelbar darunter angeordnete Graphik des österreichischen Konjunkturverlaufs (Wirtschaftswachstum) - der natürlich keine Sinusschwingung erkennen lässt - relativiert (Hitz et al. 2013, 98). Damit wird das Modellhafte der Sinusschwingung in den Vordergrund gerückt. Sehr prominent ist die Sinuskurve in Durchblick 7 (Hofmann-Schneller et al. 2015, 54) und in RGW 7 (Malcik \& Sitte 2013, 47).

Eine Darstellung der bekannten Phasen des Konjunkturverlaufs (Aufschwung, Boom, Rezession, Depression) ist überall zu finden, allerdings in unterschiedlichen Formen und unterschiedlicher Ausführlichkeit. Geospots 7 bis 8 erweitern um eine weitere Phase der Stagnation, die mit „wirtschaftspolitische Parameter bleiben über einen längeren Zeitraum auf dem gleichen Niveau, Nullwachstum" (Germ et al. 2013, 99) problematisch beschrieben wird (denn bei Nullwachstum über einen längeren Zeitraum würde man von steigender Arbeitslosigkeit und Deflationsgefahr ausgehen und könnte - wenn der Zeitraum lang genug ist - auch von Depression sprechen.) In Meridiane 7 wird noch eine sechste Phase der Erholung ausgewiesen (Hitz et al. 2013, 98). Meridiane 7 und klar_Geographie und Wirtschaftskunde verzichten dabei auf eine genauere Beschreibung der Phasen des Konjunkturverlaufs, diese sind lediglich den jeweiligen Sinuskurven zugeordnet.

Eine besondere Beschäftigung mit einer Depression ist nirgends zu finden. In klar_ Geographie und Wirtschaftskunde 7 wird der Konjunkturzyklus allerdings nach dem Kapitel „Die Finanz- und Wirtschaftskrise von 2009" behandelt - damit wird Konjunktur und Wirtschaftskrise viel unmittelbarer und konkreter eine ausführliche Beschäftigung mit den Gefahren einer Depression gibt es aber auch hier nicht (Kaps 2011, 62 ff.). In Geospots 7 bis 8 findet sich eine kurze Darstellung einer Depression, inhaltlich problematisch wird diese dabei aber als Phase hoher Inflation gesehen - eigentlich ist von Deflationsgefahr auszugehen (Germ et al. 2013, 99). In Durchblick 7 gibt es eine kurze Darstellung der Depression als massive Wirtschaftskrise, die auch politische Folgen zeitigt aber durch die Anordnung als eine Phase des Konjunkturzyklus und die Darstellung der Konjunktur als Sinuskurve entlang eines stetig steigenden langfristigen Trendwachstums geht die Dringlichkeit verloren
(Hofmann-Schneller et al. 2015, 54). In einer solchen Darstellung kann der Eindruck nicht ausgeschlossen werden, dass eine Depression einen natürlichen und periodisch wiederkehrenden Teil des Konjunkturverlaufs bildet und der nächste Aufschwung lediglich eine Frage der Zeit darstellt.

Verschärft wird dieses Problem, wenn - wie in RGW 7 - explizit ein „moderner" von einem "klassischen" Konjunkturverlauf getrennt wird und angemerkt wird, „seit 1945 sind Rezessionen nur Phasen mit einem geringeren Wachstum" (Malcik \& Sitte 2015, 47). Während eine solche Darstellung bis 2008 nachvollziehbar ist, können aktuelle Entwicklungen in dieser Form nicht gefasst werden.

\section{Vorschläge für den Unterricht}

Eine Beschäftigung mit Theorien des Konjunkturzyklus hat mehre Vorteile: Diese betonen eine Wirtschaft, die sich nicht in einem permanenten Gleichgewichtszustand befindet, sondern Krisen beinhaltet (vielleicht mit Ausnahme der neoklassischen Modelle, deren Abschwünge keine echten Krisen darstellen). Krisen werden in der Regel auch aus dem Modell intern erklärt und bedürfen nicht eines exogenen Schocks (wieder mit Ausnahme der neoklassischen Modelle). Für die Beschäftigung mit einer instabilen Welt ist das von Vorteil. Die Idee des Konjunkturzyklus lenkt den Blick auch auf die Phasen und Entwicklungen vor der Krise, was mehrschichtige Krisenerklärungen ermöglicht.

Für eine Beschäftigung mit dem Begriff des Konjunkturzyklus spricht auch, dass dieser in den allgemeinen Sprachgebrauch aufgenommen wurde und in ökonomischer Literatur ununterbrochen (und meist unhinterfragt) vorkommt. Oft bezeichnet Konjunkturzyklus einfach Wechsellagen einer Wirtschaft.

Auf der anderen Seite wurde in der Geschichte der Konjunkturtheorie gezeigt, dass die Theorien des Konjunkturzyklus dazu neigen aus der Wirtschaft einen ewigen und teilweise naturalisierten Prozess zu machen, der dem menschlichen Handeln nicht mehr zugänglich ist, anstatt einer sozialen Organisation, die von Politik verändert werden kann.

Außerdem neigen die Theorien des Konjunkturzyklus dazu alle Krisen einheitlich zu erklären. Damit werden Krisen weniger zufällig, und tatsächlich sind krisenhafte Mechanismen zu unterschiedlichen Zeiten auch ähnlich - um ein Beispiel einflussreicher neuerer Literatur zu nennen, findet etwa der Postkeynesianer Richard Koo in der Depression der 1930er Jahre und in der aktuellen Weltwirtschaftskrise ähnliche Probleme einer "balance sheet recession“ (Koo 2015). Aber Koo wendet dieses Konzept nicht auf jeden Kon- 
junktureinbruch seit 1840 an. In aktueller Literatur gibt es zahllose Versuche den Kriseneinbruch von 2007/2008 und die anhaltenden wirtschaftlichen Probleme zu analysieren (eine Zusammenstellung würde den Rahmen dieses Artikels sprengen), aber wichtige neuere Versuche jeden Kriseneinbruch der kapitalistischen Entwicklung einheitlich zu erklären, sind nicht vorhanden.

Moderne VWL-Lehrbücher sind bei der Darstellung des Konjunkturzyklus auch vor der Verarbeitung der Weltwirtschaftskrise deutlich vorsichtiger geworden. Bei Goodwin, Nelson und Harris findet sich eine stilisierte Darstellung des Konjunkturzyklus als einzelnes U mit Schultern statt einer Sinuskurve. Anstelle einer strengen Abfolge von Phasen setzen sie Expansion und Contraction, Hochpunkt und Tiefpunkt des U werden mit Peak und Through bezeichnet (Goodwin et al. 2009, 206). In der Beschreibung des Konjunkturzyklus findet man: "What economists generally do agree on is that there have been - historically a least - episodes when economies have "overheated" [...], giving rise to inflationary pressures. And there have been episodes when economies have fallen into throughs, with unacceptable levels of unemployment" (ebd., 209 f.).

Aber auch ein entschärfter Konjunkturzyklus, wie er sich bei Goodwin, Nelson und Harris findet (nicht zu verwechseln mit Richard Goodwin, dessen Konjunkturtheorie oben zusammengefasst wurde) darf über die Behandlung von Wechsellagen der Wirtschaft die Sicht auf die besondere Bedeutung der Depression nicht verstellen. Schließlich lässt sich die Wucht der aktuellen Krise durch die Darstellung eines Wechsels zwischen Boom und Rezession nur schwer fassen. Zur Veranschaulichung: Im Diagramm zur südeuropäischen Arbeitslosigkeit ist eine mehrjährige Katastrophe zu entdecken, Vollbeschäftigung gibt es aber das letzte Mal in den 1970er Jahren (Ameco 2016).

In den USA, die unter den großen Industriestaaten die Krise am besten überstanden hat, ist die Labour Force Participation Rate (Erwerbsbeteiligung) der breiteste Indikator für den Zustand des Arbeitsmarktes, von 66,4 \% im Dezember 2006 auf 62,6 \% im Dezember 2015 gesunken (Bureau of Labour Statistics 2016). Und am 1. Februar 2016 rentieren fünfährige Staatsanleihen der Eurozone im Schnitt mit -0,29 \% (Financial Times 2016). Von einer Normalisierung der Finanzmärkte kann also auch fast acht Jahre nach dem Beginn der Finanzkrise keine Rede sein.

Dies ist nur eine Auswahl an Beispielen, aber aus diesen lässt sich schließen, dass das Nachkriegsparadigma ständigen Wachstums und grundlegender wirtschaftlicher Stabilität erschüttert ist. Heutige Maturantinnen und Maturanten sind mit wirtschaftlicher Unsicherheit, erhöhter Arbeitslosigkeit und Krisenschlagzeilen der Medien konfrontiert. GW-

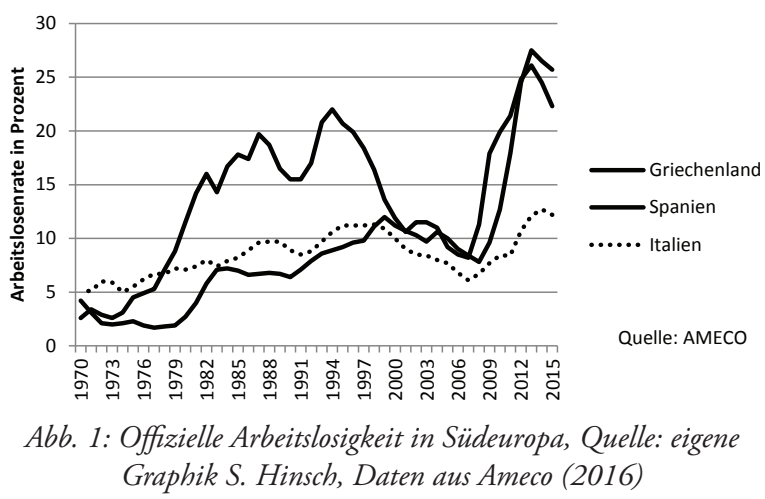

Unterricht sollte bei der Reflexion dieser persönlichen Situation helfen und zu einer Beteiligung an einer demokratischen Diskussion über Wirtschaftspolitik beitragen. Dies ist das wohl wichtigste Problem der meisten oben analysierten Schulbuchdarstellungen. Im Vordergrund steht der Zyklus, nicht das Problem der Depression. Bis zur Krise von 2007/08 ist das verständlich und vertretbar, und auch danach brauchen Schulbuchautorinnen und -autoren eine Zeit der Anpassung an neue Ansätze. Aber nach fast zehn Jahren Krise muss GW-Unterricht auf die neue Realität reagieren.

In der Darstellung der Konjunktur sei daher eine vollständige Abkehr vom Bild der Sinuskurve vorgeschlagen. Wenn eine graphische didaktische Reduktion für notwendig empfunden wird, wäre ein $\mathrm{U}$ mit Schultern besser geeignet. Sprachlich müsste bei Verwendung des Begriffs Konjunkturzyklus der Zyklus hinterfragt werden, der als solches bereits Regelmäßigkeit impliziert.

Bei den „Phasen der Konjunktur" wird eine Konzentration auf Boom und Rezession empfohlen. Schüler/innen sind durchaus in der Lage zu verstehen, dass Wirtschaftswachstum nicht nur hoch oder negativ sein kann, sondern auch Werte dazwischen annimmt - auch ohne dass solche Zustände extra ausgewiesen und definiert werden. Eine Liste von vier oder mehr Phasen impliziert wieder ein eine mögliche Regelmäßigkeit, so als müssten alle diese Phasen immer durchlaufen werden.

Andernfalls können im Unterricht auch konkrete Verläufe von Wirtschaftswachstum, Arbeitslosigkeit und Inflation gegenübergestellt werden (oder von Schülerinnen und Schülern selbst erarbeitet werden), um die Merkmale eines Booms und einer Rezession herauszuarbeiten. Das Beschreiben eines Konjunkturverlaufs, sowie das Entdecken von Zusammenhängen zwischen niedrigem Wachstum, steigender Arbeitslosigkeit, Deflationsgefahr und schwacher Nachfrage ist wichtiger als die Definition von Phasen - die in der Folge gerne zu Prüfungsstoff umgewandelt und dem Auswendiglernen und Vergessen übergeben werden. 
Und schließlich sei eine gesonderte Beschäftigung mit Depression, ihren möglichen Ursachen und ihren Auswirkungen empfohlen. Als Anregung zur Beschäftigung mit politischen Bruchlinien, die durch die aktuelle Krise aufgerissen sind, eignet sich etwa eine Analyse südeuropäischer Wahlresultate. Depressionen führen zur Krise von Wirtschaften, von volkswirtschaftlichen Paradigmen und politischen Systemen. Im GW-Unterricht muss die Depression der Regelmäßigkeit entrissen werden, sie darf nicht im Zyklus verschwinden.

\section{$4 \quad$ Literatur}

AMECO - The annual macro-economic database of the European Commission's Directorate General for Economic and Financial Affairs (2016): Unemployment. Percentage of Civilian Labour Force. http://ec.europa. eu/economy_finance/ameco/user/serie/SelectSerie.cfm (01.02.2016).

Besomi, D. (2009): Clément Juglar and his contemporaries on the causes of commercial crises. In: European Journal of Social Sciences, XLVII-143, 17-47.

Bordo, M. \& D. Wheelock, (1998): Price Stability and Financial Stability. The Historical Record. In: Federal Reserve Bank of St. Louis Review September/ October 1998, 41-62. https://research.stlouisfed.org/ publications/review/1998/09/01/price-stability-andfinancial-stability-the-historical-record/ (27.11.2015).

Bureau of Labour Statistics (2016): Civilian Labour Force Participation Rate. http://data.bls.gov/timeseries/ LNS11300000 (1.2.2016).

Debreu, G. (1959): Theory of Value. An Axiomatic Analysis of Economic Equilibrium. New Haven und London: Yale University Press. http://cowles.yale.edu/sites/ default/files/files/pub/mon/m17-all.pdf (01.02.2016).

Eichengreen, B. (2007): The European Economy since 1945. Princeton und Oxford: Princeton University Press.

Financial Times (2016): Bonds and Rate Overview. http:// markets.ft.com/Research/Markets/Bonds (01.02.2016).

Friedman, M. \& A. Schwartz (1963): A Monetary History of the United States 1867-1960. Princeton: Princeton University Press.

Frydman, R. \& M. Goldberg (2011): Beyond Mechanical Markets. Asset Price Swings, Risk, and the Role of the State. Oxford und Princeton: Princeton University Press.
Germ, A, F. Hochreiner, G. Mayerhofer \& F. Part (2013): Geospots 7 bis 8. Linz: Veritas.

Goodwin, N., J.A. Nelson \& J. Harris (2009): Macroeconomics in Context. Armonk und London: M.E. Sharpe.

Hicks, J.R. (1950): A Contribution on the Theory of the Trade Cycle. Oxford: Oxford University Press.

Hinsch, S. \& W. Langthaler (2016): Europa zerbricht am Euro. Unter deutscher Vorherrschaft in die Krise. Wien: Promedia.

Hitz, H, A. Kowarz, I. Kucera \& W. Malcik (2013): Meridiane 7. Wien: Ed. Hölzel.

Hofmann-Schneller, M., M. Deflinger, G. Menschik \& P. Rak (2015): Durchblick 7. Wien: Westermann.

Kaps, B. (2011): klar_Geographie und Wirtschaftskunde 7. Wien: Jugend und Volk.

Keynes, J.M. (1997/1936): The General Theory of Employment, Interest, and Money. Amherst: Prometheus Books.

Krugman, P. (2009): The Return of Depression Economics and the Crisis of 2008. New York und London: W. W. Norton \& Company.

Kader, M. \& U. Schwarzer (2015): Akkumulation Reloaded. Wie Notenbanken und Staaten die Profite retten. In: PROKLA 179, 2 (2015), 277-296.

Kulla, B. (1996): Die Anfänge der empirischen Konjunkturtheorie in Deutschland 1925-1933. Berlin: Duncker und Humbolt.

Maußner, A. (1994): Konjunkturtheorie. Heidelberg und Berlin: Springer Verlag.

Malcik W. \& W. Sitte (2013): Raum Gesellschaft Wirtschaft 7. Wien: Ed. Hölzel

Minsky, H.P. (2008/1986): Stabilizing an Unstable Economy. New York: McGraw-Hill.

Plossner C. (1989): Understanding Real Business Cycles. In: The Journal of Economic Perspectives 3 (3). (Summer, 1989), 51-77. http://links.jstor.org/sici?sici=08953309\%28198922\%293\%3A3\%3C51\%3AURBC\%3E2 .0.CO\%3B2-Q (15.01.2016)

Schumpeter, J. (1939): Business Cycles. A Theoretical, Historical and Statistical Analysis of the Capitalist Process. New York: McGraw-Hill. http://classiques. uqac.ca/classiques/Schumpeter_joseph/business_cycles/ schumpeter_business_cycles.pdf (15.01.2016).

Sowell, T. (2006): On Classical Economics. Yale: Yale University Press. 